\title{
A Fault Simulation Analysis of Sensor Networks Based on Wavelet Analysis
}

\author{
Shutao Guo ${ }^{1,}$, Dejing Cui ${ }^{1, b}$, Binguo Wang ${ }^{2}$, Tinglei Zhang ${ }^{2}$ \\ ${ }^{1}$ Hengde Digital Choreography Technology Co., Ltd, Qingdao Economic and \\ Technological Development Zone, Shandong, China \\ ${ }^{2}$ College of Information Science and Engineering, Shandong University of Science and Technology, \\ Qingdao, Shandong, China \\ ahengdewm@126.com, bcuidejing836@163.com
}

\begin{abstract}
Keywords: Sensor technology, The fault diagnosis analysis, Continuous wavelet analysis and Matlab simulation

Abstract. The rapid development of modern information technology to drive the development of sensor technology has become one of the pillars of modern information technology development. The reliability of the sensors as sensor applications in various industries is more and more attention. Therefore, sensor fault diagnosis technology is more and more importance. This paper takes the digital stage integrated information collection system inertial sensor as an example, and it not only introduces the application of wavelet analysis in sensor fault diagnosis, but also studied the application function of continuous wavelet transforms. Through simulation experiment, we simulated and verified the effect of continuous wavelet transforms in the sensor fault. The experiment proved that the continuous wavelet transform analyses have good sensitivity and anti - noise in sensor fault diagnosis, then it shows that the wavelet transform analysis method has a good practical application in sensor network fault.
\end{abstract}

\section{Introduction}

Sensor fault modes have uncertainty ${ }^{[1]}$ and diversified forms. Based on this characteristic, we need to make effective classification for sensor fault in research of the sensor fault, in order to make classificatory analysis from different problems direction in the research process. There is conducive to propose more and more effective analysis methods. Fault classifications have several different ways:

(1) The degree of fault (hard faults and soft faults);

(2) Fault performance characteristics (permanent faults and intermittent faults);

(3) The process of fault stage (slowly varying fault and sudden fault);

(4) Modeling angle (addition and multiplication fault);

(5) The cause of the fault (error fault, shock fault, periodic fault, short circuit fault).

Wavelet analysis ${ }^{[2]}$ is a gradually developing emerging discipline since the 1990s. Wavelet transform analysis is a multi-resolution analysis method. The basic idea of wavelet analysis is used a family of functions to represent or approximate a signal or function. It has time domain decay and frequency domain decay, volatility characteristics.

Signal have a very important feature, which is the more important information is often irregular mutations and singular point mutations of the signal. Signal mutation is an important manifestation of sensor failure, which also provides basis of research and detection for fault diagnosis. For a long time, we study the singularity of function by studying the Fourier Transforms ${ }^{[3]}$. The method works by attenuation of function in the Fourier transform domain to obtain singularity characteristics of function and calculate the singularity value. However, the Fourier transform has its inherent flaws, that is, it can only determine the integrity singular characteristic of a function and lack region singular characteristic. Which also determines Fourier transform cannot calculate spatial location and distribution of singular points. The wavelet transforms spatial locality will determine its analysis results better then Fourier transforms. Therefore, according to be obtained the singularity characteristic of signals by wavelet analysis, we can analysis and detection to get the time point and degree of sensor malfunction. Below we detail introduction the fault simulation analysis of sensor based on wavelet transforms. 


\section{The theoretical basis of wavelet analysis}

In order to better research fault simulation detection method of wavelet analysis, we first introduce the basis mathematical theoretical of wavelet analysis. Set $\Psi(t) \in L^{2}(R)$, Fourier transform can be expressed as $\hat{\Psi}(w)$, if the condition is satisfied:

$$
\int_{0}^{+\infty} \frac{|\hat{\Psi}(w)|^{2}}{w} d w=\int_{-\infty}^{0} \frac{|\hat{\Psi}(w)|^{2}}{w} d w=C_{\Psi}<+\infty
$$

Then, there is a mother wavelet function $\Psi(t)$, this function is square inferable.

According to the wavelet transform theory ${ }^{[4]}$, common mother wavelets have Daubechies(dbN), Haar, Mexican Hat(mexh), Coiflet(coifN), Biorthogonal(biorNr.Nd), Morlet(morl), Meyer, Symmlets(symN) and so on.

Continuous wavelet transforms. The mother wavelet function $\Psi(t)$ be scaled and translated to obtain wavelet basis function:

$$
\Psi_{a, b}(t)=\frac{1}{\sqrt{|a|}} \Psi\left(\frac{t-b}{a}\right), a, b \in Z
$$

Among, a represents the scale factor, $\mathrm{b}$ represents the displacement factor. Due to the scale factor a and displacement factor values are taken the value of continuously changing, so called $\Psi_{\mathrm{a}, \mathrm{b}}(\mathrm{t})$ is a continuous wavelet basis function. If $\mathrm{f}(t) \in L^{2}(R)$, then, the continuous wavelet transform of $\mathrm{f}(\mathrm{t})$ can be defined as follows:

$$
\Psi_{f}(a, b)=|a|^{-\frac{1}{2}} \int_{-\infty}^{+\infty} f(t) \Psi\left(\frac{t-b}{a}\right) d t
$$

Its inverse transformation can be expressed as:

$$
f(t)=\frac{1}{C_{\Psi}} \int_{-\infty}^{\infty} \int_{-\infty}^{\infty} \frac{1}{a^{2}} W_{f}(a, b) \Psi\left(\frac{t-b}{a}\right) d a d b
$$

Discrete wavelet transforms. Linked to the practical application and combined with the specific operation in computer simulation ${ }^{[4]}$, we discrete the continuous wavelet. Here, we discuss the discretization of continuous wavelet functions $\Psi_{a, b}(t)$ and continuous wavelet transform $W_{f}(a, b)$. The wavelet transform is not a time variable discrete, but it is discretization of continuous translation parameter and continuous scale parameters. With signal on the time axis discrete has essentially different.

Among the continuous wavelet transform,

$$
\Psi_{a, b}(t)=\frac{1}{\sqrt{|a|}} \Psi\left(\frac{t-b}{a}\right)
$$

Here, $b \in R, a \in R_{+}$, and $a \neq 0, \Psi$ is admissibility. For ease of calculation, constrained $a$ only be positive in discrete, compatibility condition is corresponding also into:

$$
C_{\Psi}=\int_{0}^{\infty} \frac{|\widehat{\Psi}(\bar{w})|^{2}}{|\bar{w}|} d \bar{w}<\infty
$$

Thus, the coefficients of discrete wavelet transform can be expressed as follows:

$$
C_{j, k}=\int_{-\infty}^{\infty} f(t) \Psi_{j, k}(t) d t=\left\langle f, \Psi_{j, k}\right\rangle
$$

So, the corresponding reconstruction formula is transformed into:

$$
f(t)=C \sum_{-\infty}^{\infty} \sum_{-\infty}^{\infty} C_{j, k} \Psi_{j, k}(t)
$$

Where, $\mathrm{C}$ represents signal independent invariants. 


\section{The examples of Sensor fault simulation}

Wavelet transform analysis is an analytical method based on frequency analysis and time signal analysis. Wavelet transform have good locality characteristics in both time domain and frequency domain. Thus, wavelet analysis has been widespread concern and research in the field of sensor fault diagnosis based on the locality characteristics of frequency-domain and time-domain ${ }^{[5]}$.

The basic steps of simulate fault detection the based on wavelet transform analysis are:

First, the output and input signal of sensor system are continuous wavelet transform;

Second, obtain singular values of the two signals use the transform;

Third, singled out the mutations extreme point of input, and get the extreme point corresponding to the sensor fault.

In this paper, we simulation the steps of fault diagnose by Matlab software platform. We use a single input-output linear system sensor to simulation experiment, and the sensor fault can be manifested by system parameters change in this system. That

$$
\mathrm{Y}(\mathrm{s})=\mathrm{G}(\mathrm{s}) \mathrm{U}(\mathrm{s})+\triangle \mathrm{G}(\mathrm{s}) \mathrm{U}(\mathrm{s})+\mathrm{E}(\mathrm{s})
$$

Among: *(s) is a Laplace transform of the corresponding signal function; $Y(s) 、 U(s) 、 E(s)$ are the input signal, output signal and the noise corresponding signal function. Suppose E(s) is a stationary random noise and it mean is zero; $\mathrm{G}(\mathrm{s})$ is the transfer function of the sensor system; $\triangle \mathrm{G}(\mathrm{s})$ represents a system parameter variation caused by a fault-made. In order to facilitate the calculations, assumptions:

(l) $\mathrm{u}(\mathrm{t})$ represents a known stationary random signal, the signal is segmented signal, its faults and mutations occur in different time periods;

(2) $\mathrm{G}(s)$ means that when a fault occurs, the existence extreme points of $\mathrm{G}(s) \neq 0$.

Here, preliminary theorem and approximate relationship as the theoretical basis of fault detection analysis.

Preparation Theorem 3.1: Existence a stationary random signal $\mathrm{x}(\mathrm{t})$, when the mean of wavelet transform $W_{s}(s, t)$ is zero, its variance will approaches zero as increase of the scale s.

Preparation Theorem 3.2: For single-input single-output sensor system, when there is no fault, $W_{y}(s, t) \approx K * W_{u}(s, t)$ was incorporated in the s factor of the larger scale. Here, $K=G\left(w_{s 0}\right), G\left(w_{s 0}\right)$ is center frequency of $\hat{\Psi}_{s}(s, t)$. In a specific application, using the least-squares solution

$$
\begin{gathered}
\text { of } J=\min _{K} \sum_{t}\left[W_{y}(s, t)-K W_{u}(s, t)\right]^{2} \\
\hat{k}=\frac{\sum W_{y}(s, t) W_{u}(s, t)}{\sum\left[W_{y}(s, t)\right]^{2}}
\end{gathered}
$$

to replace $\mathrm{K}$.

\section{Wavelet transform of Matlab platform for sensor fault simulation and analysis}

In this paper, we use 27 blocks inertial navigation sensors for scene motion capture in stage integrated system, and we use the extreme points of continuous wavelet transform to diagnosis analyze the sensor fault.

The following experimental procedure is achieved at Matlab8.0 environment. Use Simulink simulation tools ${ }^{[6]}$ are provided by Matlab software to graphical modeling. Simulink simulation tools provide practical wavelet analysis toolkit. According to the toolkit, we can easily be modeling analysis of sensor fault diagnosis.

Assumes the sensor model of input signal $\mathrm{u}(\mathrm{t})$ mean value is not constant equal to zero, as follows:

Using a single input-output system model: $G(s)=\frac{c s+d}{s^{2}+a s+b}$ 
When the sensor is normal, $a=10, b=24, c=3, d=8$. Use mutation of system parameters to simulate mutations failure. When $\mathrm{t}=140$, a sensor failure is assumed, $\mathrm{c}$ mutated into $5, \mathrm{~d}$ mutated into 20 , the rest coefficient input does not happen change. Input signal value $u(t)$ is a random signal, the signal evenly distributed, its mean mutation occurs at $t=65 \mathrm{~s}$, from 0.5 to 2 .

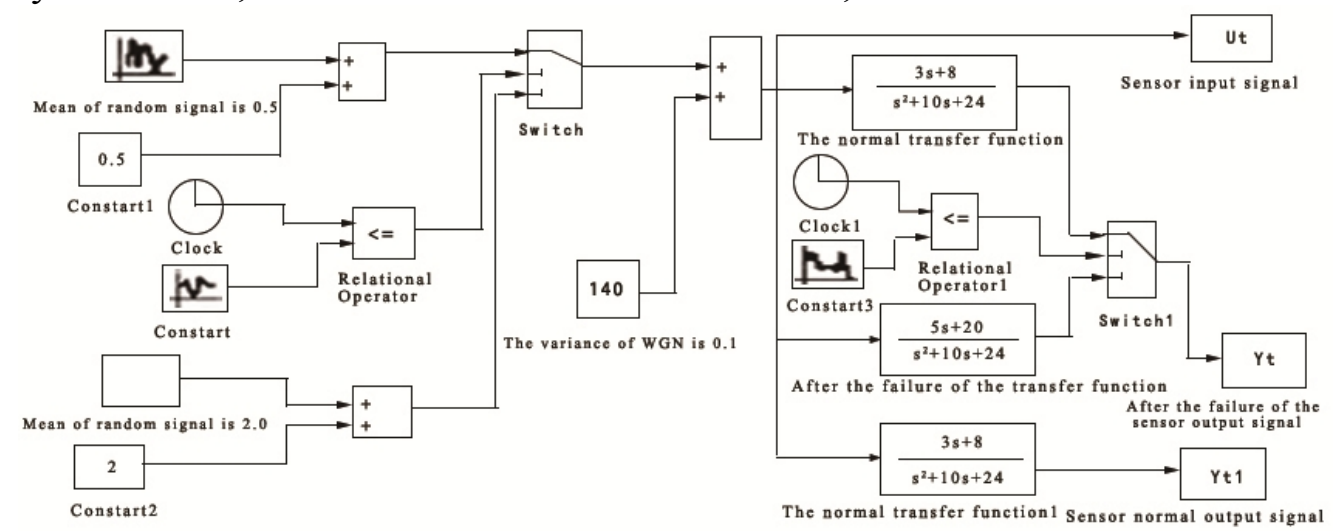

Figure 1 Fault diagnosis system model

According to the above method, the diagnoses are as follows:

The first step, using simulation environment to establish fault diagnosis system model in matlab, see Figure 1, assuming the sampling time is $0.03 \mathrm{~s}$, run the program to diagnostic: $\mathrm{t}=0: 0.01: 150$;

Coefl $=\operatorname{cwt}\left(U t,[2,4,8],{ }^{\prime} d b 3^{\prime}\right) ; \quad \% U t$ is a sensor input signal;

Coef2 $=\operatorname{cwt}\left(Y t 1,[2,4,8],{ }^{\prime} d b 3^{\prime}\right) ; \%$ Ytl indicates no fault output signal;

Coef $3=\operatorname{cwt}\left(\mathrm{Y} t,[2,4,8],{ }^{\prime} d b 3^{\prime}\right) ; \quad \% \mathrm{Yt}$ is output signal when the fault occur;

$\%$ when $\mathrm{a}=2,4,8$, compute extreme value;

for $\mathrm{i}=1: 3$

$\mathrm{kl}=\operatorname{sum}(\operatorname{coef}(\mathrm{i},:) * \operatorname{coef} 2(\mathrm{i},:)) ; \quad \%$ calculated the molecular moiety in Formula (3.2);

$\mathrm{k} 2=\operatorname{sum}\left(\operatorname{coefl}(\mathrm{i},:) .{ }^{*} \operatorname{coefl}(\mathrm{i},:)\right) ; \%$ calculated the denominator in Formula (3.2);

$\mathrm{k}=\mathrm{k} 1 / \mathrm{k} 2 ; \quad \%$ calculated the value of $\hat{k}$ in Formula (3.2);

$\mathrm{Rtt}=\operatorname{coef} 3(\mathrm{i},:)-\mathrm{k} * \operatorname{coef}(\mathrm{i},:) ; \quad \%$ calculated the value of $r(t)$ in Formula (3.3);

$\mathrm{Rt}=\left[\mathrm{t}^{\prime}, \mathrm{R} \mathrm{tt}^{\prime}\right]$ :

$\operatorname{Subplot}(3,1, \mathrm{i}): \operatorname{plot}(\operatorname{Rt}(:, 1), \operatorname{Rt}(:, 2)) ; \operatorname{Xlabel}\left(\left[{ }^{\prime} \mathrm{a}={ }^{\prime}, \operatorname{num} 2 \operatorname{str}\left(2,{ }^{\wedge} \mathrm{i}\right)\right],{ }^{\prime} \operatorname{Extremal}\right.$ graph is under $\left.)\right)$ end

\section{Simulation Experiments}

According to the diversity of sensor failure, refer to the engineering experience and failures, and classification of inertial navigation sensor in the application environment to analyze the reasons of faults formation for these types, shown in Table 1

Table 1 The reasons of faults formation

\begin{tabular}{|l|l|}
\hline Fault Type & Fault Cause \\
\hline Bias fault & bias voltage and bias current, etc \\
\hline Impact interference & $\begin{array}{l}\text { machinery sparks, the burr of converter, random interference of earth } \\
\text { wire and power, etc }\end{array}$ \\
\hline Short-circuit fault & short circuit $\backslash$ bridge corrosion caused by electrochemical pollution, etc \\
\hline Open-circuit fault & $\begin{array}{l}\text { Welding Weld, signal line is disconnected, sealing off, chip pin } \\
\text { disconnection, etc }\end{array}$ \\
\hline Drift fault & zero drift, sensitivity drift, temperature drift, etc \\
\hline Periodic interference fault & 50HZ power co-channel interference, etc \\
\hline Nonlinear with Dead zone & Links with nonlinear, amplifier saturation, etc \\
\hline
\end{tabular}


Their computer simulation realization methods are as follows:

(1) Bias fault: adding a constant small signal output signal;

(2) Impact interference: Add a pulse signal on the output signal;

(3) Short-circuit fault: the sensor output signal infinity approaching to zero;

(4) open-circuit fault: the output signal approaching to the maximum amount of output value;

(5) Drift fault: output signal is offset based on a constant rate;

(6) Periodic interference fault: superimposed signal of a given frequency;

To study the sensor fault diagnosis simulation analysis using continuous wavelet transform extreme points described in Section 3. According to Section 3, when $t=140$ s simulating the mutation of extreme figure, which is the fault. However, due to a larger sampling time, there is more interference in other time periods. The noise caused larger error in the extreme figure, so fault detection difficult. Therefore, increasing the sampling time, wavelet diagnosis, get the following diagram, see Figure 2:

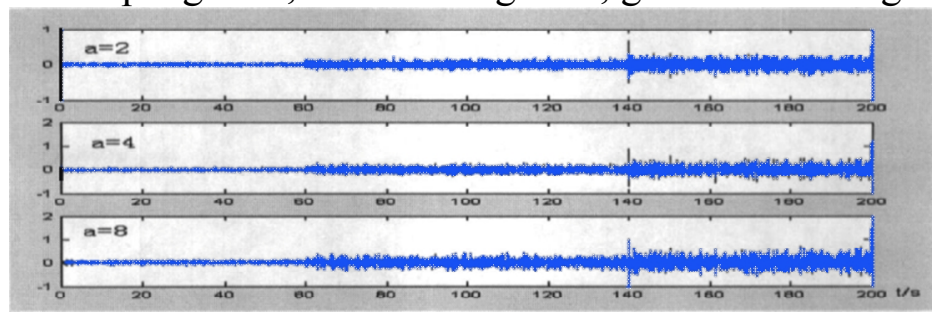

Figure 2 Extremum image

According to Figure 2, in the 140s, the obvious peak appeared in each scale. While continuing to increase the sampling frequency, to some extent, we can detect the existence of fault points. The reason for this phenomenon is that there is no process of the input signals, and generates noise. Assuming failure sampling time is $0.001 \mathrm{~s}$, the output signal and the input signal is smooth processed 50 points to get the image is shown below:

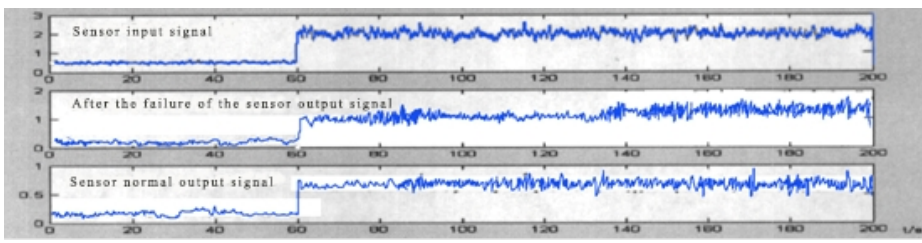

Figure 3 Extremum image

We processed input and output signals and use the above-described fault diagnosis. When using db3 wavelet, after smoothing the output and input signal, it has a good suppression interference of other time periods in extremes map of each scales. In 140s, each segment of data noise is noticeable. When the scale value is increased, the analysis result can be obtained more obvious. The above analysis shows that the theoretical basis is real and effective. Thought the input signal exist mutation information, it can effectively analyze the mutation changes condition of sensor fault.

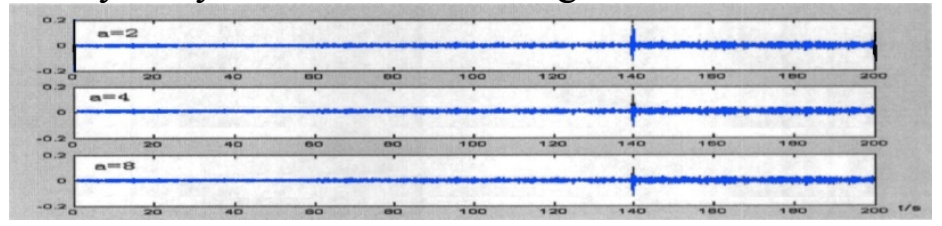

Figure 4 Extremum image

Figure 4 shows at $\mathrm{t}=140 \mathrm{~s}$, the continuous wavelet transforms appear apparent mutation characteristics. With the increasing of scale values, the value of the wavelet transforms the speed of decay to accelerate in other time period, resulting in reduced noise. So, the more powerful explanation that there is a sensor faults at this point. This is a strong proof of the wavelet transforms fault detection method is reasonable. In this paper, the experiment results show that information capture sensor failure analysis of the integrated system on the stage with signal input requirements have low correlation characteristics, high sensitivity, and good analysis results. 


\section{Conclusions}

This paper introduces the basic theory of continuous wavelet transforms and discrete wavelet transforms and its application in sensor fault diagnosis field. Combining inertial navigation sensors used in digital stage integrated system it introduced the classification of sensor fault detection and detection method of fault classification. The principle of wavelet transforms is described in detail. According to Matlab simulation platform, we use Simulink development environment for sensor fault continuous wavelet transforms real environment simulation. The simulation results show that the effect of detection sensor is well and it achieve practical requirements. Sensor failure analysis method based on wavelet analysis is rapid, simple, effective, high diagnostic accuracy, the sensor system with lower data requirements. Therefore, Sensor failure analysis method based on wavelet analysis has real value and research significance in the development of digital integration.

\section{Acknowledgment}

The authors are grateful for the support from National Key Technology R\&D Program (2013BAH60F01).

\section{References}

[1] Yanbin Zhu. Recent advances and market opportunities of transducer technology, Journal of Transducer Technology, 2000, 19(3):1-4.

[2] Wang Suiyu, Xing You, Cai Yuan. Study on the on-line fault diagnosis for power transformer based on wavelet analysis. Electronic Test, 2014, (1): 4-6

[3] Li Hongru, Feng Guoying, Du Yongzhao. Wavefront measurement based on windowed Fourier transform by use of radial shearing interferometer. High Power Laser and Particle Beams, 2013, 25(8): 1897-1903

[4] Rongbiao Zhang, Lan Cai, Guicheng Wang. Study of PH Sensor Fault Diagnosis Based on Time Series and Expert System, Journal of Electronic Measurement and Instrument, 2001, 15(3): 30-34

[5] Yuguang Li, Mingguang Liu. Using wavelet to enhance infrared image in motor fault detection, Computer Engineering and Applications, 2013, 49(11):241-243

[6] Suyama.K. Fault deteetion of redundant sensor susedin reliable sample-data control systems[C].Proeeedings of the 37th IEEE Conference on deision and control, Volume: 1, 1998.Page(s): 1161-1164 